\title{
Guide to preclinical models used to study the pathophysiology of idiopathic intracranial hypertension
}

\author{
Zerin Alimajstorovic $\mathbb{1}^{1} \cdot$ Connar S. J. Westgate $\mathbb{D}^{1,2} \cdot$ Rigmor H. Jensen $^{2} \cdot$ Sajedeh Eftekhari ${ }^{2} \cdot$ James Mitchell $^{1}$. \\ Vivek Vijay ${ }^{1}$ - Senali Y. Seneviratne $\mathbb{D}^{1} \cdot$ Susan P. Mollan ${ }^{3} \cdot$ Alexandra J. Sinclair ${ }^{1,3,4,5}$
}

Received: 13 August 2019 / Revised: 24 October 2019 / Accepted: 29 November 2019 / Published online: 2 January 2020

(c) The Author(s), under exclusive licence to The Royal College of Ophthalmologists 2020

\begin{abstract}
Idiopathic intracranial hypertension (IIH) is characterised by raised intracranial pressure (ICP) and papilloedema in the absence of an identifiable secondary cause typically occurring in young women with obesity. The impact is considerable with the potential for blindness, chronic disabling headaches, future risk of cardiovascular disease and marked healthcare utilisation. There have been marked advances in our understanding the pathophysiology of IIH including the role of androgen excess. Insight into pathophysiological underpinnings has arisen from astute clinical observations, studies, and an array of preclinical models. This article summarises the current literature pertaining to the pathophysiology of IIH. The current preclinical models relevant to gaining mechanistic insights into IIH are then discussed. In vitro and in vivo models which study CSF secretion and the effect of potentially pathogenic molecules have started to glean important mechanistic insights. These models are also useful to evaluate novel therapeutic targets to abrogate CSF secretion. Importantly, in vitro CSF secretion assays translate into relevant changes in ICP in vivo. Models of CSF absorption pertinent to IIH, are less well established but highly relevant and of future interest. There is no fully developed in vivo model of IIH but this remains an area of importance. Progress is being made to improve our understanding of the underlying aetiology in IIH including the characterisation of disease biomarkers and their mechanistic role in driving disease pathology. Preclinical models, used to evaluate IIH mechanisms are yielding important mechanistic insights. Further work to refine these techniques will provide translatable insights into disease aetiology.
\end{abstract}

Alexandra J. Sinclair

a.b.sinclair@bham.ac.uk

1 Metabolic Neurology, Institute of Metabolism and Systems Research, College of Medical and Dental Sciences, University of Birmingham, Birmingham B15 2TT, UK

2 Department of Neurology, Danish Headache Centre, Rigshospitalet-Glostrup, Glostrup Research Institute, Valdemar Hansens Vej 5, 2600 Glostrup, Denmark

3 Birmingham Neuro-Ophthalmology, Queen Elizabeth Hospital, Birmingham, UK

4 Centre for Endocrinology, Diabetes and Metabolism, Birmingham Health Partners, Birmingham B15 2TH, UK

5 Department of Neurology, University Hospitals Birmingham NHS Foundation Trust, Queen Elizabeth Hospital, Birmingham B15 2WB, UK

\section{Overview}

Idiopathic intracranial hypertension (IIH) is a neurological disorder characterised by papilloedema, in the absence of an identifiable secondary cause on neuroimaging (including venous sinus thrombosis); with an elevated lumbar puncture opening pressure and normal cerebrospinal fluid (CSF) constituents [1]. IIH typically affects obese women between the ages of 15 and 45 [2].

Headache is the commonest reported symptom which for the majority has a migrainous phenotype which significantly impacts on quality of life [3, 4]. Other commonly reported symptoms include visual manifestations such as transient visual obscurations in $68 \%$ of cases $[5,6]$, diplopia as a result of cranial nerve VI palsy [7], pulsatile tinnitus, dizziness, and back and neck pain [2]. A cardinal feature of $\mathrm{IIH}$ is the presence of papilloedema (swelling of the optic disc secondary to raised ICP) [8]. The pathophysiological basis for the appearance of papilloedema is thought to be secondary to axoplasmic stasis resulting from impaired 
transport due to raised intracranial pressure (ICP) [9]. Papilloedema can lead to visual loss which can necessitate emergency surgical intervention [10] and is reported to cause visual loss in up to $25 \%$ of cases $[11,12]$.

Clinical approaches to management are variable internationally but recently (2018) the first consensus guidelines have outlined a standardised approach to investigation and management of adult IIH $[1,10,13]$. Whilst treatment options are limited in choice and efficacy, there are now an increasing number of randomised clinical trials in this disease area [5, 14-17].

This review article is based on previously conducted studies identified by searches on the electronic databases of States National Library of Medicine (PubMed), Medline and the Cochrane Library, from listing until 1st April, 2019. The following descriptors were used to include articles for review: IIH; pseudotumour cerebri; pathophysiology; aetiology; intracranial pressure; preclinical models; CSF secretion; animal models. The articles were limited to those in English language. Abstracts were reviewed and relevant full articles were obtained but case reports and letters to the editor were excluded. Only preclinical models were included as clinical models are out of scope and not included in this review. Reference lists from articles were also examined for further citations.

The aim of this review is to inform the readership of the current literature pertaining to pathophysiological mechanisms identified in IIH and how preclinical models are allowing the field to gain mechanistic insights into IIH. Ultimately this may allow identification of targeted therapeutics to be developed.

\section{Epidemiology of IIH}

Previously the incidence of IIH was estimated at between 0.5 and 1.0 per 100,000 people [2], however, the incidence has been increasing. The largest cohort study to date of 23,182 new IIH cases reported a $108 \%$ increase from 2.26 per 100,000 in 2002 to 4.69 per 100,000 in 2016 [18]. The highest incidence was seen in females aged 25 years and was 15.2 per 100,000 . More recently a UK populationbased matched cohort study demonstrated a 350\% increased incidence over the last decade to an incidence of 9.0 per 100,000 in 2019 [19]. Increased incidence is rising in line with increased obesity rates [18]. Obesity has long been an associated risk factor for typical IIH [2], and incidence of IIH has been noted to reflect country specific obesity rates [20].

IIH has a significant financial impact. IIH costs were estimated at \$444 (£345.5) million in the United States in 2011 [21]. Recently health economic evaluation of UK hospital costs incurred from IIH were reported to rise from
Table 1 Summary of the top ten priorities for IIH research.

\begin{tabular}{ll}
\hline Top 10 priorities for IIH research \\
\hline 1 & Underlying causes of IIH \\
2 & Mechanisms of headache \\
3 & New treatments for IIH \\
4 & Predicting visual loss \\
5 & Monitoring visual function \\
6 & IIH biomarkers \\
7 & Hormones \\
8 & Headache management \\
9 & Understanding the relationship to weight and weight loss \\
10 & Optimal intervention \\
\hline
\end{tabular}

$£ 9.2$ (\$12.3) million in 2002 to $£ 49.9$ (\$64.0) million in 2014 , and projected costs to UK hospital services at $£ 462.7$ (\$593.6) million by 2030 [18]. Rising costs are predominantly driven by high hospital attendance rates, as well as scanning and procedure rates, as well as indirect costs such as absence from work or reduced work capacity.

There are many unanswered questions in IIH. Patients and professionals have voiced their opinions on the research priorities in IIH through a James Lind Alliance Research priority setting partnership. Identifying the aetiology of IIH was placed as the number one priority [22] (Table 1).

The pathophysiology of IIH has been speculated for over a century and many causative factors have been postulated [23-25]. There have been a number of key advances over the last decade with a focus on identifying disease specific biomarkers. IIH biomarkers may represent either cause or consequence of the disease and their validity and interpretation is enhanced though understanding their mechanistic role. Preclinical models are a useful tool to enhance our understanding of IIH disease pathophysiology and underpinning mechanisms. The number of preclinical models is growing and their relevance, utility and interpretation requires careful scrutiny.

Here we aim to update the current thinking on IIH pathophysiology and provide a guide to the preclinical models which evaluate CSF secretion, CSF absorption and animal models pertinent to IIH.

\section{Pathophysiology overview}

The pathophysiology of IIH has been speculated over time. Due to elevated ICP much of the literature surrounding the disease has focussed on hypersecretion of CSF and/or a reduction of CSF absorption through the arachnoid granulations or the glymphatic system [26]. To understand central nervous system fluid dynamics, a basic understanding of normal anatomy and physiology is important. There are 


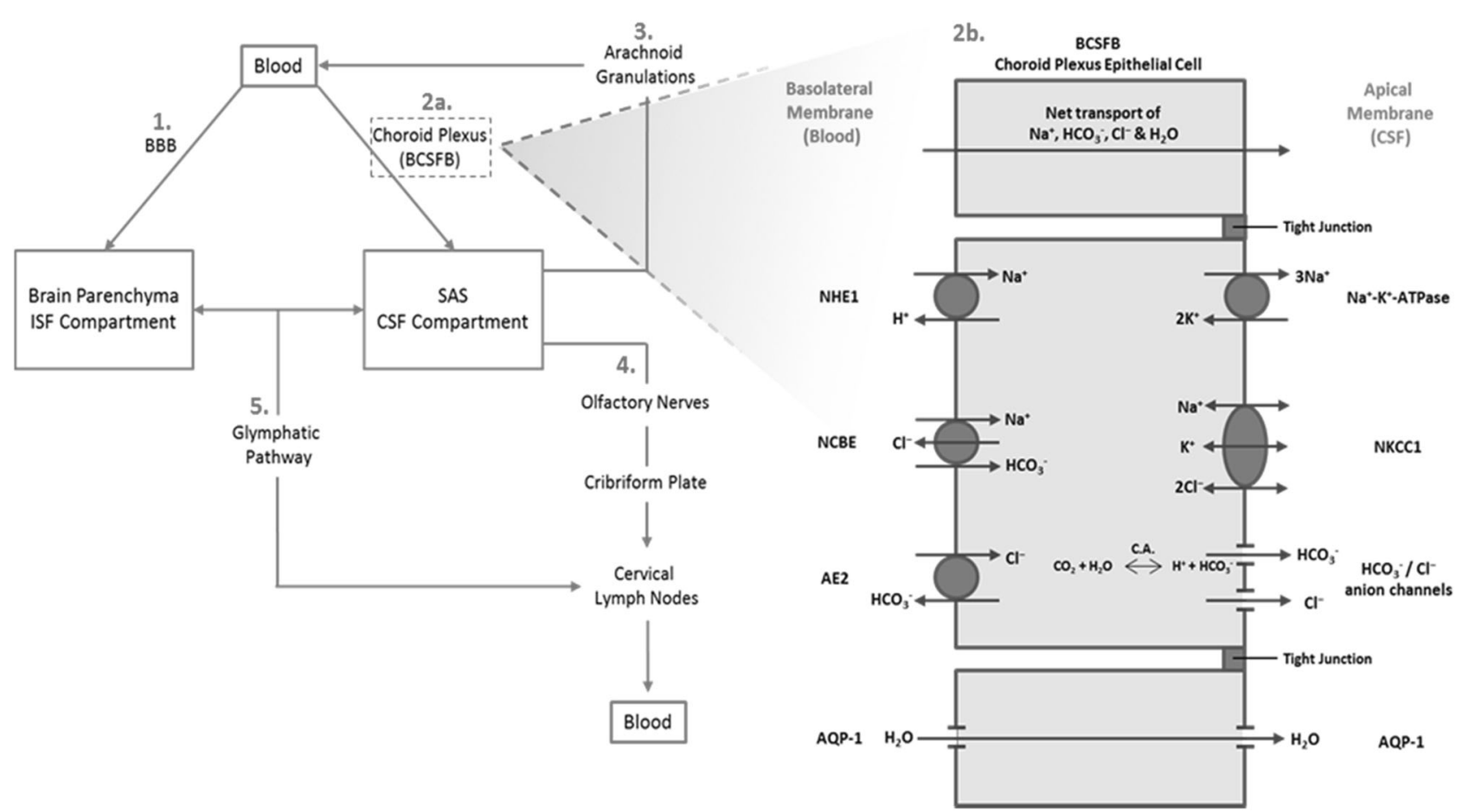

Fig. 1 Schematic diagram of main transport proteins of CSF secretion within choroid plexus epithelia and the putative pathway of CSF secretion and absorption. The diagram shows the CSF and ISF movement between the brain barriers from secretion to absorption of both fluids. Fluid movement is shown by the blue arrows between the BBB (brain capillary endothelium) (1); BCSFB (CP epithelium) (2a) into the brain compartments and absorption through the arachnoid barrier (3) back into the blood. Evagination of the arachnoid membrane into the lumen of the vein and therefore the dural vessel allows the communication between CSF in the SAS and blood. CSF also passes through the SAS to the olfactory bulbs and then drains into nasal lymphatics by passing through the cribriform plate and along channels adjacent to olfactory nerves and into the blood (4). The

three main barrier sites between the brain and the blood: the blood-brain barrier, the blood-CSF-barrier and the arachnoid epithelium as shown in Fig. 1.

\section{CSF secretion}

Approximately $80 \%$ of CSF is produced by the epithelial cells of the choroid plexus, organs that reside in the cerebral ventricles, with $20 \%$ being secreted by the ventricular ependymal and the brain tissue itself $[27,28]$. The functional unit of the choroid plexus is the choroid plexus villus, which comprises of central fenestrated capillaries surrounded by choroid plexus epithelial cells, where through the formation of tight junctions, the choroid plexus villus constitutes the blood-CSF-barrier and controls CSF composition.

Choroid plexus epithelial cells have critical roles related to directed transport processes from blood into the CSF, exchange of fluid between the CSF in the SAS and the ISF in the brain parenchyma allows these fluids to drain into the cervical lymph nodes through the glymphatic pathway (5). The highlighted region (2b) of the diagram depicts the $\mathrm{CP}$ with the net transport of $\mathrm{Na}^{+}, \mathrm{HCO}_{3}{ }^{-}, \mathrm{Cl}^{-}$ and water, from basolateral (blood) to apical (CSF) membranes, with schematic locations of ion transporters $\mathrm{Na}^{+}-\mathrm{K}^{+}$-ATPase, NKCC1, $\mathrm{HCO}^{3-}$ and $\mathrm{Cl}^{-}$channels are localised to the apical CSF facing membrane. NHE1, NCBE and AE2 transporters are expressed on the basolateral blood facing membrane. AQP-1 water channels are expressed on both sides of the $\mathrm{CP}$ epithelium. Blood-brain barrier (BBB), blood cerebrospinal fluid barrier (BCSFB), carbonic anhydrase (CA), choroid plexus (CP), interstitial fluid (ISF), subarachnoid space (SAS).

removal of substances out of the brain, and for CSF production [27]. The pressure required for the circulation of CSF is maintained by a hydrostatic pressure gradient between the choroid plexus (highest pressure at site of CSF formation) and the arachnoid granulations (lowest pressure at site of CSF absorption) [29].

The total volume of CSF within adult humans is $\sim 140 \mathrm{ml}$ and the rate of secretion by each choroid plexus is $0.2 \mathrm{ml} /$ $\min [27,30]$ or $500 \mathrm{ml}$ per day [31].

Fluid secretion by choroid plexus epithelial cells depends on the unidirectional transport of ions, facilitated by transport proteins that are found in the apical (lumen) and basolateral (blood) membranes of the cells [28] (Fig. 1), generating an osmotic gradient which induces net water movement [32]. Fluid secretion by these cells involves the sodium-potassium ATPase pump $\left(\mathrm{Na}^{+}-\mathrm{K}^{+}\right.$-ATPase), epithelial sodium channel, chloride channel (CIC-2) and aquaporin-1 water channel (AQP-1) located on the apical membrane, driving the efflux of $\mathrm{Na}^{+}, \mathrm{Cl}^{-}, \mathrm{K}^{+}, \mathrm{HCO}_{3}{ }^{-}$and 
$\mathrm{H}_{2} \mathrm{O}$ into the CSF [27]. Chloride-bicarbonate exchanger (AE2) and sodium-bicarbonate co-transporters (NCBn1, $\mathrm{NCBE}$ ), are located on the basolateral membrane and drive the accumulation of $\mathrm{Na}^{+}, \mathrm{Cl}^{-}$and $\mathrm{HCO}_{3}{ }^{-}$into the cytoplasm of the choroid plexus epithelial cells, eventually resulting in CSF secretion [27].

However, conventional osmotic forces may not account for the known production rate of CSF and the molecular mechanisms underlying CSF production remain under investigation [31]. The osmotic processes are being facilitated by AQP-1, and studies have revealed that AQP-1 knockout mice decrease CSF secretion and ICP but do not fully ameliorate CSF secretion [33]. Studies have reported the novel concept of the cotransport of water involving the $\mathrm{Na}^{+}-\mathrm{K}^{+}-2 \mathrm{Cl}^{-}$cotransporter (NKCC1), contributing to approximately half of the CSF production [31]. This suggests NKCC1 transporters action upon water and CSF secretion works independently of AQP-1 and explains why AQP-1 knockout does not fully prevent CSF secretion [31, 34].

\section{In vitro-models of CSF secretion}

In vitro CSF secretion assays fall into two generic groups; those that directly assess CSF secretion and those that assess surrogates of CSF secretion.

Porcine choroid plexus cultures have been previously (1998) utilised to assess CSF secretion in vitro and has the potential to screen molecules that alter CSF secretion [35]. However, pigs are not standard model organisms. The assay has not been reliably replicated in other centres. Rodent choroid plexus does not provide sufficient material in primary cultures to make such an assay ethically viable, or increase throughput due to the amount of animals that need to be sacrificed.

As such $\mathrm{Na}^{+}-\mathrm{K}^{+}$-ATPase activity at the choroid plexus is utilised in vitro as a surrogate of CSF secretion [36-39], and the physiological rationale behind this is clear. Inhibiting $\mathrm{Na}^{+}-\mathrm{K}^{+}$-ATPase inhibits CSF secretion in vivo by up to $80 \%$ of baseline, thus $\mathrm{Na}^{+}-\mathrm{K}^{+}$-ATPase activity is intrinsically linked to CSF secretion [39]. These assays directly assess ionic movement, and ATPase activity. Importantly, these models are predictive of the in vivo effects of drugs [38, 40]. However, these assays only measure end points and thus are prone to variability. More recently a novel $\mathrm{Na}^{+}-\mathrm{K}^{+}$-ATPase activity assay assessed ATPase activity in real-time through live cell imaging, and reduced variability provided data comparable to other assays [41].

As NKCC1 channels have been demonstrated to contribute to CSF secretion [31], assessing the activity of the NKCC1 channel, and other channels proposed to directly transport water, would allow a more complete understanding of how molecules alter CSF secretion [42]. But the impact of each of the myriad of ion channels on overall CSF production is not fully understood.

\section{In vivo models of CSF secretion}

The main aim of in vivo models is to measure CSF secretion rates within physiological parameters. However, a number of models have been studied previously and are highlighted in Table 2.

In summary, CSF secretion rate experiments are interpreted by the classic hypothesis of CSF hydrodynamics [43]. The advantages of these techniques include marker substances such as Dextran can only be diluted by newly formed CSF in the case of the ventriculo-cisternal perfusion technique [43]. The aqueductal method allows for a closed system, which permits the CSF pressure between the ventricular and subarachnoid spaces in a physiological range [44, 45]. However, in most cases the techniques used are not performed under physiological conditions (normal CSF secretion rate: $7 \mu \mathrm{l} / \mathrm{min}$ in cats [45], $2 \mu \mathrm{l} / \mathrm{min}$ in rats [46]), which can limit their interpretation. Also, air and CSF absorption from the CSF has to be prevented [43]. Therefore, a novel method is necessary to determine the physiology and pathophysiology of CSF dynamics.

\section{CSF absorption}

There are three main routes of CSF absorption: arachnoid granulations, nasal and dural lymphatics and the glymphatic system [47-51].

\section{CSF absorption by arachnoid granulations}

Each choroid plexus secretes CSF into the ventricle in which it is situated. The ependymal epithelium, which lines the ventricles, is in contact with the CSF. The CSF and the interstitial fluid of the brain parenchyma are separated by the perivascular space that surrounds the large blood vessels (Figs. 2 and 3) [49, 52]. There is a constant directional flow of CSF from the cerebral ventricles to the subarachnoid space. The openings of the subarachnoid space into the dural venous system through arachnoid granulations have classically thought to be the primary site of CSF absorption back into the blood [2, 27, 29, 53]. Hydrostatic pressure is maintained in the subarachnoid space allowing the flow of CSF into the dural sinus. As the opening is valvular, the blood cannot cross in the opposite direction [54, 55].

Although debated, venous sinus stenosis has been reported to be present in over $90 \%$ of $\mathrm{IIH}$ patients; resulting in 


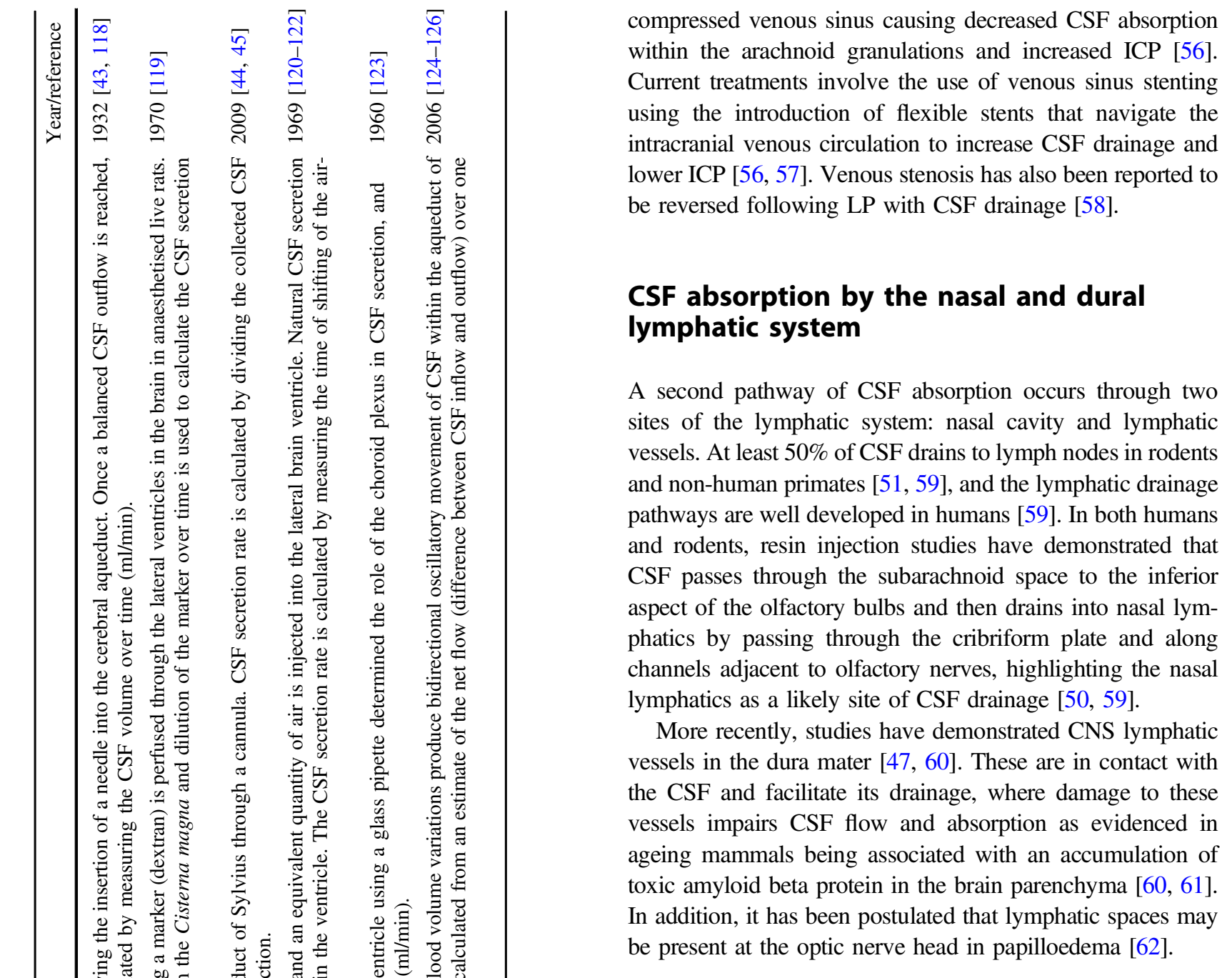

CSF absorption by the glymphatic system

A third route of fluid clearance through paravenous drainage pathways involves the exchange of fluid between the CSF in the subarachnoid space and the interstitial fluid in the brain parenchyma [49]. These glial lymphatics or 'glymphatics' facilitate the passive movement of both CSF and interstitial fluid from the cranium to the periphery through a pressure gradient, facilitated by aquaporin-4 on astrocytes [63, 64].

The relative contribution of each of these pathways to normal physiology and in the disease state of IIH is unknown.

\section{In vitro-models of CSF absorption}

In vitro assessment of CSF absorption is an under developed area with few dedicated assays available. In vitro and ex vivo CSF absorption showing unidirectional fluid movement from the apical to basolateral aspects has been 
demonstrated in cadaveric human arachnoid granulation tissue $[65,66]$. Such an assay has the potential to screen for molecules that alter CSF absorption at the arachnoid granulations. However, the rarity of human arachnoid granulation tissue for culture and the absence of arachnoid granulations in rodents make such an assay impractical as a routine laboratory technique.

The association of other structures, such as the nasal and dural lymphatics with CSF drainage, presents the possibility to utilise assays to assess fluid transports across a monolayer of lymphatic endothelial cells to provide a surrogate assay for CSF drainage. In addition, the demonstration that the glymphatic system contributes to CSF absorption suggests that

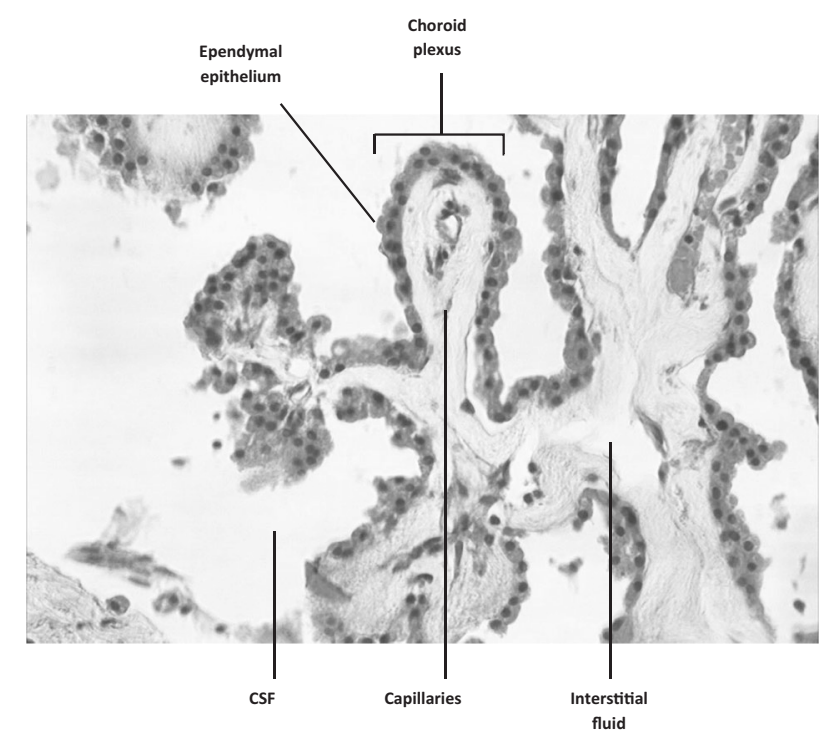

Fig. 2 Human choroid plexus H\&E stained $(\times 40)$.

A

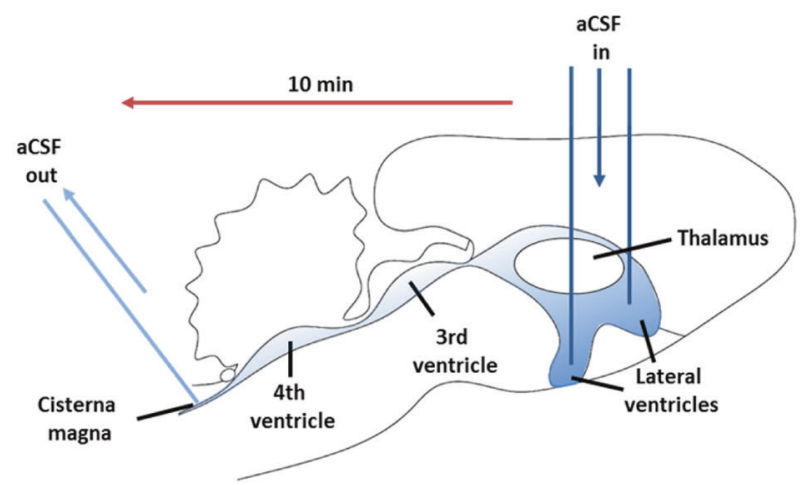

Fig. 3 Adult rat brain and the site of injection into the lateral ventricle for ventriculo-cisternal perfusion and variable rate infusion. a Ventriculo-cisternal perfusion: aCSF (containing blue dextran) was perfused into each lateral ventricle of the rat brain. The solutions were collected every $10 \mathrm{~min}$ from the Cisterna magna and the absorbance was read on a spectrophotometer. The increase in dilution of the blue dextran within the aCSF as it passes through each ventricle indicates an increase in CSF secretion over the course of assessing in vitro fluid transport in cultured astrocytes could provide a surrogate for glymphatic CSF absorption [49]. The above assays exist and as such should be explored, providing a potential screening mechanism that could complement in vivo assessment of glymphatic CSF drainage [67].

\section{In vivo-models of CSF absorption}

Few models of CSF absorption have been studied previously, as described in Table 3, but are essential in determining the mechanism upon which CSF is drained from the brain under physiological parameters (normal CSF pressure: $11.9 \mathrm{cmH}_{2} \mathrm{O}$ in cats [68]; $9.5 \mathrm{cmH}_{2} \mathrm{O}$ in rats [69]).

The variable rate infusion technique is useful to study the effects of mediators upon resistances to CSF absorption but is particularly invasive. However, both imaging techniques are less invasive and are useful tools to study CSF lymphatic absorption in order to understand this pathway in CNS disease models [48].

\section{Effects of obesity and gender on CSF dynamics in IIH}

Obesity and female gender are known risk factors of IIH [23]. Studies by Newborg were the first to document diet as a treatment for IIH in open label case series [70]. Variable weight loss occurred in following a low calorie and low sodium diet, and resulted in improvement in IIH symptoms and papilloedema [70]. Other studies found a weight loss of $6 \%$ in female IIH patients with obesity resulted in recovery of

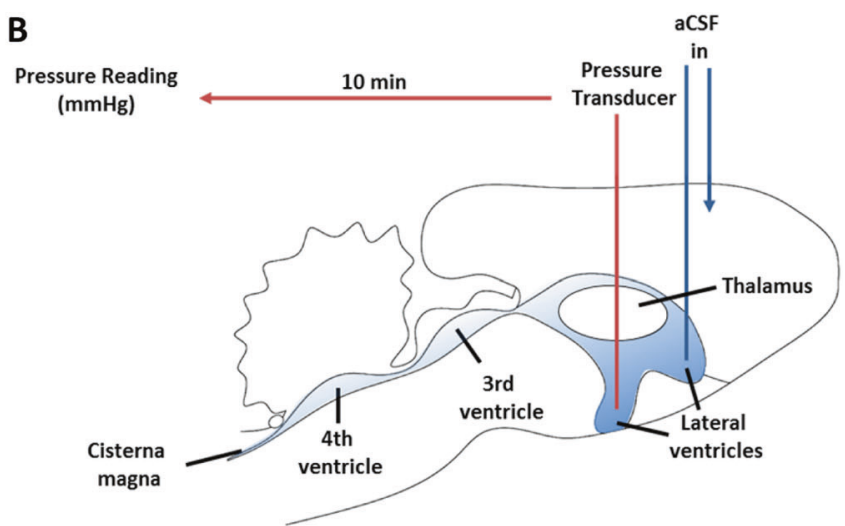

experiment. b Variable rate infusion: aCSF was perfused into one lateral ventricle of the rat brain at increasing perfusion rates. A separate cannula, attached to a pressure transducer, was inserted into the other lateral ventricle and pressure readings were taken every $10 \mathrm{~min}$. The increase in dilution of the blue dextran within the aCSF for each sample indicates an increase in CSF secretion over the course of experiment; therefore as it is a closed system this would lead to an increase in pressure. Artificial cerebrospinal fluid (aCSF). 
Table 3 In vivo models of CSF absorption.

\begin{tabular}{|c|c|c|}
\hline Method & Description & Year/Reference \\
\hline Variable rate infusion & $\begin{array}{l}\text { Infusion of aCSF into the lateral ventricle at a known flow rate causes CSF pressure to } \\
\text { rise to a plateau level. The resistance to CSF absorption }\left(\mathrm{mmH}_{2} \mathrm{O} \text { min/ } \mu \text { l) is calculated }\right. \\
\text { from the gradient of CSF pressure at plateau level against the infusion rate (Fig. } 3 \mathrm{~b}) \text {. }\end{array}$ & $1987[127]$ \\
\hline $\begin{array}{l}\text { Hyperspectral imaging of lymphatic } \\
\text { drainage }\end{array}$ & $\begin{array}{l}\text { Quantum dot } 655 \text { fluorescent particles are injected into the CSF of the Cisterna magna } \\
\text { and visualised by in vivo hyperspectral imaging at varying time points. A quantum dot } \\
\text { signal, detected in submandibular lymph nodes, determines the site of CSF absorption. }\end{array}$ & 2013 [48] \\
\hline High resolution stereomicroscopy & $\begin{array}{l}\text { Lymphatic-reporter mice characterised the anatomical routes and dynamics of CSF } \\
\text { outflow. This non-invasive imaging technique quantified the transport of tracers to the } \\
\text { blood and lymph nodes following injection into the lateral ventricles. }\end{array}$ & $2017[128]$ \\
\hline
\end{tabular}

papilloedema and visual field dysfunction in $74 \%$ of cases [71]. Re-evaluation after 3 months, following maintenance of a low energy diet with no evidence in weight gain, showed that reduced ICP and papilloedema were maintained [72]. Reducing weight is considered the optimal approach to decrease ICP and modify the underlying disease in IIH $[1,2,72]$.

Obesity and female gender have also been suggested to impair CSF absorption pathways [26, 73]. Some studies have noted reduced CSF absorption in those with IIH [73]. Other studies used isotope methods which evaluated CSF circulation and absorption, and cisternographic findings to show an increased arachnoid resistance to CSF efflux might be present in those with IIH [74].

Further studies suggested thrombosis in the arachnoid granulations, increased thrombotic gene expression, and increased resistance to CSF absorption through inflammation of the arachnoid granulations in female IIH patients is potentially aggravated by thrombophilic exogenous oestrogens [75]. The risk factor of prothrombotic effects may be hypothesised to cause increased resistance to CSF absorption and consequently elevated ICP but this has not been substantiated.

\section{Hormones and IIH}

Retinoids (vitamin A), steroid hormones such as corticosteroids and sex steroids have all been hypothesised to alter CSF dynamics [26].

\section{Retinoids}

Studies have described the intake of large quantities of vitamin A causing secondary intracranial hypertension symptoms [76-78] and vitamin A pharmacological therapies have been associated with elevated CSF pressure in adults [79]. Indeed IIH patients have been identified to have altered retinoid metabolism by several studies [80, 81]. However, a definitive evaluation conducted using samples from the Idiopathic Intracranial Hypertension Treatment Trial, which was noted for its accurate quantitative assessment of vitamin A metabolites, demonstrated that although IIH patients have mild abnormalities in retinoid metabolism, these abnormalities were not altered following resolution of symptoms suggesting that vitamin A toxicity is unlikely to contribute to the aetiology of IIH [78].

\section{Glucocorticoids}

Glucocorticoids (GCs) are pleotropic steroid hormones where cortisol is the major physiologically active GC in humans. It has previously been demonstrated that, following weight loss in IIH patients, reductions in the global capacity to activate GCs through reduction in $11 \beta$ hydroxysteroid dehydrogenase type 1 (11 $\beta$-HSD1), is associated with reduced ICP [82]. This coupled with the expression of $11 \beta$-HSD1, the enzyme that gates tissue level activation of GCs, at choroid plexus epithelial cells, suggests that GCs could modulate ICP [83]. Indeed, elevated levels of cortisol in the choroid plexus epithelium driven by $11 \beta$-HSD1 drives apical $\mathrm{Na}^{+}$channels to increase movement of $\mathrm{Na}^{+}$and therefore water molecules into the CSF, thereby driving CSF secretion and ICP [82, 84].

Of interest, in an embryologically related tissue, the ocular ciliary body, where the mechanisms of fluid secretion (aqueous humour) is analogous to that of CSF secretion at the choroid plexus, demonstrated inhibition of 11 $\beta$-HSD1 $[84,85]$. Of note, activity of $11 \beta$-HSD1 in patients with IIH correlates significantly with ICP changes. However, there are a lack of human studies evaluating the systemic and central nervous system GC phenotype in IIH patients compared with matched controls.

\section{Leptin}

Leptin is an adipokine that circulates in serum at levels proportionate to body fat and modulates appetite and energy balance $[86,87]$. Leptin is a product of the Obese $(\mathrm{Ob})$ gene 
and when absent, results in profound obesity in rodents and humans [88, 89]. Leptin elicits its action centrally in the hypothalamus where leptin is delivered via the blood-brain barrier and the CSF, where the organ responsible for leptin transport in the CSF is the choroid plexus [90].

Due to the association of IIH and obesity, leptin levels have been assessed in both the serum and CSF in IIH patients [86, 91-94]. There is however no consensus as to the leptin phenotype in IIH due to conflicting study designs (Table 4). However, the majority of studies reported increased serum leptin, and the largest study assessing CSF leptin reported increased CSF leptin in IIH. In addition, this study demonstrated that CSF leptin levels positively correlate with lumbar puncture opening pressure in non-IIH patients, suggesting leptin could modulate ICP [86]. Indeed, chronic leptin exposure increases $\mathrm{Na}^{+}-\mathrm{K}^{+}$-ATPase activity in renal tubule cells $[95,96]$. As $\mathrm{Na}^{+}-\mathrm{K}^{+}$-ATPase activity increases CSF secretion at the choroid plexus, it may be hypothesised that leptin increases CSF secretion; however, there is currently no direct evidence to support this theory.

\section{Oestrogen and progesterone}

Given that IIH is predominantly a disease of women, the levels of the female sex hormones progesterone and oestrogens have been assessed. Studies of these sex hormones and IIH demonstrate inconclusive results, confounded by small cohorts and use historical assays $(n=5-51$ IIH patients, 1991-2013) [26, 97, 98]. These studies were also hampered through the use of radioimmunoassay, which are noted to have low reliability, a poor lower limit of sensitivity as well as issues with cross reactivity of some metabolites leading to unspecificity [99].

Historical case series evaluating the use of hormonal contraceptives have suggested a possible causal role in $\mathrm{IIH}$ through several case studies [26]. However a recent longitudinal meta-analysis $(n=53)$ suggests that the use of hormonal contraceptives confers no additional risk to the development of IIH [100]. It should also be noted that oral contraceptives are a hugely heterogenous group of medications. They differ in the oestrogen effects, sex hormone binding globulin effects and progestogenic effects. In some the progestogen components have mild anti-androgenic effects; others have mild proandrogenic effects while others have direct anti-androgenetic effects. The net effect is also further complicated by differing dosages used across populations. Thus, case series analyses that evaluate the effect of hormonal contraceptives as a whole in $\mathrm{IIH}$, without consideration for contraceptive subtype cannot be reliably interpreted.

Animal experimentation suggest both oestrogen and progesterone decrease $\mathrm{Na}^{+}-\mathrm{K}^{+}$-ATPase activity thus potentially
Table 4 Leptin levels in serum and CSF of IIH patients compared with controls.

\begin{tabular}{lllll}
\hline Leptin in IIH & Numbers & Matching & Fasted & Year/Study \\
\hline$\uparrow$ Serum & IIH 15 & BMI: Yes & Fasted & 2002 \\
N/A CSF & Control 16 & Age: No & {$[91]$} \\
$\uparrow$ Serum & CSF: 22 IIH, 56 control & BMI: No & Non-fasted & 2009 \\
$\uparrow C S F$ & Serum: 26 IIH, 53 control & Age: No & {$[86]$} \\
- Serum & $8 \mathrm{IIH}$ & BMI: Yes & Non-fasted & 2009 \\
- CSF & 8 controls & Age: Yes & {$[92]$} \\
$\uparrow S e r u m$ & IIH 10 & BMI: No Non-fasted & 2010 \\
- CSF & Control 20 & Age: Yes & {$[93]$} \\
-Serum & 36 IIH & BMI: Yes & Fasted & 2017 \\
N/A CSF & 40 controls & Age: Yes & {$[94]$} \\
\hline
\end{tabular}

reduce CSF production [101]. However, the direct effect of these hormones on ICP has yet to be established.

\section{Androgens}

The importance of androgens in the pathophysiology of IIH has recently come to the fore following the discovery that $\mathrm{IIH}$ is characterised by a unique androgen excess signature which modulates CSF dynamics.

Androgens are sex steroids that are primarily generated in ovarian thecal cells and activated peripherally by the skin, liver and adipose tissue [102]. The cardinal disease of female androgen excess, polycystic ovary syndrome (PCOS) is prevalent in IIH with some case series reporting up to $57 \%$ of patients presenting with PCOS [75, 103]. In female-to-male gender reassignment patients, testosterone therapy has been suggested to cause raised ICP and papilloedema which goes into remission temporally with testosterone withdrawal [104106]. Indeed, young onset ( $<25$ years of age) female IIH patients are associated androgen excess [98].

Steroid metabolite analysis using liquid chromatography-tandem mass spectrometry was performed in serum, urine and CSF of patients with IIH $(n=70)$, PCOS $(n=60)$ and in patients with simple obesity $(n=40)$ matched for age, gender and BMI. IIH women showed a pattern of androgen excess distinct to that observed in PCOS and simple obesity, with increased serum testosterone, and increased CSF testosterone and androstenedione. 11-oxygenated androgens, which are adrenal derived and a major contributor to androgen excess in PCOS, were not deranged in IIH [107]. In addition, specific to IIH, systemic steroid metabolism measured in $24 \mathrm{~h}$ urine samples using gas chromatography-mass spectrometry demonstrated a net increase in systemic $5 \alpha$-reductase activity, an important reaction in androgen activation [107].

The study also demonstrated that IIH was uniquely characterised by elevated CSF levels of the active androgen testosterone as well as its precursor androstenedione [107]. 
Table 5 Advantages and disadvantages of in vivo ICP monitoring models.

\begin{tabular}{|c|c|c|c|}
\hline Model & Advantages & Disadvantages & Year/Study \\
\hline Epidural ICP probe & $\begin{array}{l}\text { - Minimally invasive } \\
\text { - Mid-long term } \\
\text { monitoring ( } 30 \text { days) }\end{array}$ & $\begin{array}{l}\text { - Sedated during reading } \\
\text { - Anaesthesia could alter ICP }\end{array}$ & $2017[36,40]$ \\
\hline $\begin{array}{l}\text { Rat intraventricular } \\
\text { cannula model }\end{array}$ & $\begin{array}{l}\text { - Freely awake animals } \\
\text { - Mid-long term } \\
\text { monitoring ( } 15 \text { days })\end{array}$ & $\begin{array}{l}\text { Highly invasive technique } \\
\text { - Possible inflammation/ } \\
\text { infection/primary } \\
\text { tissue damage }\end{array}$ & $\begin{array}{l}2013,1987 \\
{[129-131]}\end{array}$ \\
\hline Telemetric animal system & $\begin{array}{l}\text { - Freely awake animals } \\
\text { - Wireless } \\
\text { - Long term monitoring } \\
\text { (>8 weeks) }\end{array}$ & $\begin{array}{l}\text { - Highly invasive technique } \\
\text { - Possible inflammation/ } \\
\text { infection/primary } \\
\text { tissue damage } \\
\text { - Cannot work in mice } \\
\text { - Expensive } \\
\text { - Long recovery time ( } 7 \text { days) } \\
\text { following implantation }\end{array}$ & $\begin{array}{l}\text { 2016, 2011, } \\
2015[132-134]\end{array}$ \\
\hline
\end{tabular}

The choroid plexus epithelial cells express the androgen receptor as well as the key enzymatic machinery for androgen metabolism. Furthermore testosterone has the capacity to increase $\mathrm{Na}^{+}-\mathrm{K}^{+}$-ATPase activity in rodent choroid plexus cells, a surrogate marker of CSF secretion [36] suggesting testosterones capacity to increase ICP, thus potentially pathogenicity in IIH [41]. Interestingly, systemic androgen production is reduced following weight loss in IIH patients and concurrent resolution of symptoms [82]. This suggests that adipose tissue could be in part driving the androgen excess phenotype, but this has yet to be assessed.

Androgen excess is a key driver of increased cardiovascular risk [108-113]. In a population-based matched cohort study patients with IIH were found to have doubled risk of cardiovascular disease compared to age, gender and BMI matched control subjects [19]. This supports a concept that IIH is a complex disease characterised by systemic metabolic dysregulation. Interestingly, increased centripetal fat drives the risk of metabolic syndrome and cardiovascular disease [114-116]. IIH is characterised by a centripetal fat distribution which correlates with ICP [117].

\section{Moving towards an in vivo IIH model}

Obesity in rodents has been postulated to model IIH. Although there are several available models of rodent obesity, to our knowledge only one has assessed the role of obesity with regards to ICP. Obese Zucker rats, who have a dysfunctional leptin receptor, have raised ICP when compared with lean controls [40]. However, the ICP recordings of over fed rats, that develop obesity over 4 weeks, showed a tendency to correlate positively with weight gain, but this did not reach significance [40]. ICP recordings were achieved using an epidural ICP probe thereby utilising minimally invasive in vivo ICP monitoring and suggests that obesity could elevate ICP [40]. The advantages and disadvantages of this technique as well as other in vivo models of ICP are described in Table 5.

Thus assessing the capacity of other models of obesogenesis should be assessed with regards to ICP. Other models of obesity need to be explored.

\section{Conclusion}

This paper has explored the current pathophysiological mechanisms identified in IIH. Due to the nature of this descriptive review there are inherent limitations in the methodology, for example it did not critically grade each article for bias, and only those published in English have been included. It does however serve to provide the clinician with an in-depth understanding of the complex processes that are now thought to have influence on our patients. The last decade has seen major advances in understanding IIH. Systemic metabolic dysregulations may be a key causal driver in disease pathogenesis with a unique androgen excess profile recently identified in IIH and implicated in CSF dysregulation. A twofold increase in cardiovascular disease risk in IIH further supports reclassifying IIH as a systemic disease. Mechanistic studies, through the use of preclinical models, are vital to further advance the understanding of IIH. An in vivo model to fully explain IIH has yet to be established and in the interim the use of preclinical models provide a fundamental resource to facilitate mechanistic evaluations as well as evaluation of potential novel therapeutics. However, these models all have clear pros and cons which are vital to grasp in order to critically interpret the ensuing study results.

DH I National Institute for Health Research (NIHR)NIHR-CS-011-028 [Sinclair]. 
RCUK | Medical Research Council (MRC)-MR/ $\mathrm{K} 015184 / 1$.

\section{Summary}

\section{What was known before}

- The aetiology of IIH has long been speculated.

- The role of increased CSF secretion or decreased CSF drainage as a cause of elevated ICP is debated.

- Although biomarkers have been identified in IIH, studies are small and have previously lacked a mechanistic evaluation.

- There are limited in vitro and in vivo models of IIH to evaluate underlying mechanisms in IIH.

\section{What this study adds}

- A unique androgen excess profile which modulates CSF dynamics has been identified in IIH.

- Androgens modulate CSF secretion via the choroid plexus, affecting ICP regulation, and are highlighted as the most important therapeutic target in IIH.

- In vitro and in vivo models of CSF dynamics are emerging and growing in sophistication in their ability to inform regarding underlying aetiology in IIH.

- Understanding the pros and cons of these preclinical models is vital to enable accurate interpretation of the ensuing results.

- There is currently no in vivo model to fully explain IIH.

Funding AS is funded by the National Institute of Health Research (NIHR) UK (NIHR Clinician Scientist Fellowship (NIHR-CS-011028), to AJS) and the Medical Research Council UK (project grant MR/K015184/1).

\section{Compliance with ethical standards}

Conflict of interest The authors declare that they have no conflict of interest.

Publisher's note Springer Nature remains neutral with regard to jurisdictional claims in published maps and institutional affiliations.

\section{References}

1. Mollan SP, et al. Idiopathic intracranial hypertension: consensus guidelines on management. 2018;89:1088-100.

2. Markey KA, et al. Understanding idiopathic intracranial hypertension: mechanisms, management, and future directions. Lancet Neurol. 2016;15:78-91.
3. Mulla Y, et al. Headache determines quality of life in idiopathic intracranial hypertension. J Headache Pain. 2015;16:521.

4. Mollan SP, Hoffmann J, Sinclair AJ. Advances in the understanding of headache in idiopathic intracranial hypertension. Curr Opin Neurol. 2019;32:92-8.

5. Wall M, et al. The idiopathic intracranial hypertension treatment trial: clinical profile at baseline. JAMA Neurol. 2014;71:693-701.

6. Julayanont $\mathrm{P}$, et al. Idiopathic intracranial hypertension: ongoing clinical challenges and future prospects. J Pain Res. 2016;9:87-99.

7. Mollan SP, et al. A practical approach to, diagnosis, assessment and management of idiopathic intracranial hypertension. Pract Neurol. 2014;14:380-90.

8. Wall M, George D. Idiopathic intracranial hypertension. A prospective study of 50 patients. Brain. 1991;114:155-80.

9. Mackenzie P, Cioffi G. How does lowering of intraocular pressure protect the optic nerve? Surv Ophthalmol. 2008;53(Suppl 1): S39-43.

10. Mollan SP, et al. Evaluation and management of adult idiopathic intracranial hypertension. Pract Neurol. 2018;18:485.

11. Best $\mathrm{J}$, et al. The incidence of blindness due to idiopathic intracranial hypertension in the UK. Open Ophthalmol J. 2013;7:26-9.

12. Corbett JJ, et al. Visual loss in pseudotumor cerebri: follow-up of 57 patients from five to 41 years and a profile of 14 patients with permanent severe visual loss. Arch Neurol. 1982;39:461-74.

13. Hoffmann J, et al. European headache federation guideline on idiopathic intracranial hypertension. $\mathrm{J}$ Headache Pain. 2018;19:93.

14. Ball AK, et al. A randomised controlled trial of treatment for idiopathic intracranial hypertension. J Neurol. 2011;258:874-81.

15. Smith SV, Friedman DI. Idiopathic intracranial hypertension treat trial: a rev outcomes. Headache. 2017;57:1303-10.

16. Markey KA, et al. Assessing the efficacy and safety of an 11 betahydroxysteroid dehydrogenase Type 1 inhibitor (AZD4017) in the Idiopathic Intracranial Hypertension Drug Trial, IIH:DT: clinical methods and design for a phase II randomized controlled trial. JMIR Res Protoc. 2017;6:e181.

17. Ottridge $\mathrm{R}$, et al. Randomised controlled trial of bariatric surgery versus a community weight loss programme for the sustained treatment of idiopathic intracranial hypertension: the Idiopathic Intracranial Hypertension Weight Trial (IIH:WT) protocol. BMJ Open. 2017;7:e017426.

18. Mollan SP, et al. The expanding burden of idiopathic intracranial hypertension. Eye. 2018;33:478-85.

19. Adderley NJ, et al. Association between idiopathic intracranial hypertension and risk of cardiovascular diseases in women in the United Kingdom. JAMA Neurol. 2019;76:1088-98.

20. McCluskey G, et al. Meta-analysis and systematic review of population-based epidemiological studies in idiopathic intracranial hypertension. Eur J Neurol. 2018;25:1218-27.

21. Friesner D, et al. Idiopathic intracranial hypertension in the USA: the role of obesity in establishing prevalence and healthcare costs. Obes Rev. 2011;12:e372-80.

22. Mollan SHK, Herd Clare, Denton A, Williamson M, Sinclair Alexandra. Research priorities for idiopathic intracranial hypertension. BMJ Open. 2019;9:e026573.

23. Hornby $\mathrm{C}$, et al. Metabolic concepts in idiopathic intracranial hypertension and their potential for therapeutic intervention. $\mathrm{J}$ Neuroophthalmol. 2018;38:522-30.

24. Markey KA, et al. Idiopathic intracranial hypertension, hormones, and 11beta-hydroxysteroid dehydrogenases. J Pain Res. 2016;9:223-32.

25. Sinclair AJ, et al. Exploring the pathogenesis of IIH: an inflammatory perspective. J Neuroimmunol. 2008;201-202C:212-20. 
26. Mollan SP, et al. Evolving evidence in adult idiopathic intracranial hypertension: pathophysiology and management. J Neurol Neurosurg Psychiatry. 2016;87:982-92.

27. Brown PD, et al. Molecular mechanisms of cerebrospinal fluid production. Neuroscience. 2004;129:957-70.

28. Hladky SB, Barrand MA. Fluid and ion transfer across the bloodbrain and blood-cerebrospinal fluid barriers; a comparative account of mechanisms and roles. Fluids Barriers CNS. 2016;13:19.

29. Oreskovic D, Klarica M. Measurement of cerebrospinal fluid formation and absorption by ventriculo-cisternal perfusion: what is really measured? Croat Med J. 2014;55:317-27.

30. Speake T, et al. Mechanisms of CSF secretion by the choroid plexus. Microsc Res Tech. 2001;52:49-59.

31. Steffensen AB, et al. Cotransporter-mediated water transport underlying cerebrospinal fluid formation. Nat Commun. 2018;9:2167.

32. Oreskovic D, Rados M, Klarica M. Role of choroid plexus in cerebrospinal fluid hydrodynamics. Neuroscience. 2017;354:69-87.

33. Oshio K, et al. Reduced cerebrospinal fluid production and intracranial pressure in mice lacking choroid plexus water channel Aquaporin-1. Faseb J. 2005;19:76-8.

34. Hamann $\mathrm{S}$, et al. Cotransport of water by the $\mathrm{Na}+-\mathrm{K}+-2 \mathrm{Cl}(-)$ cotransporter NKCC1 in mammalian epithelial cells. J Physiol. 2010;588:4089-101.

35. Hakvoort A, Haselbach M, Galla HJ. Active transport properties of porcine choroid plexus cells in culture. Brain Res. 1998;795:247-56

36. Botfield HF, et al. A glucagon-like peptide-1 receptor agonist reduces intracranial pressure in a rat model of hydrocephalus. Sci Transl Med. 2017;9:1-11.

37. Damkier HH, Brown PD, Praetorius J. Cerebrospinal fluid secretion by the choroid plexus. Physiol Rev. 2013;93:1847-92.

38. Klarr SA, et al. The effects of hypo- and hyperkalemia on choroid plexus potassium transport. Brain Res. 1997;758: 39-44.

39. Vates TS Jr., Bonting SL, Oppelt WW. NA-K activated adenosine triphosphatase formation of cerebrospinal fluid in the cat. Am J Physiol. 1964;206:1165-72.

40. Uldall $\mathrm{M}$, et al. Choroid plexus aquaporin 1 and intracranial pressure are increased in obese rats: towards an idiopathic intracranial hypertension model? Int J Obes. 2017;41:1141.

41. O'Reilly MW, et al. A unique androgen excess signature in idiopathic intracranial hypertension is linked to cerebrospinal fluid dynamics. JCI Insight. 2019;4:125348.

42. MacAulay N, Zeuthen T. Water transport between CNS compartments: contributions of aquaporins and cotransporters. Neuroscience. 2010;168:941-56.

43. Orešković D, Klarica M. The formation of cerebrospinal fluid: nearly a hundred years of interpretations and misinterpretations. Brain Res Rev. 2010;64:241-62.

44. Oreskovic D, Klarica M, Vukic M. The formation and circulation of cerebrospinal fluid inside the cat brain ventricles: a fact or an illusion?. Neurosci Lett. 2002;327:103-6.

45. Klarica M, et al. New experimental model of acute aqueductal blockage in cats: effects on cerebrospinal fluid pressure and the size of brain ventricles. Neuroscience. 2009;158:1397-405.

46. Pardridge WM. Drug transport in brain via the cerebrospinal fluid. Fluids Barriers CNS. 2011;8:7.

47. Louveau A, et al. Structural and functional features of central nervous system lymphatic vessels. Nature. 2015;523:337-41.

48. Mathieu $\mathrm{E}$, et al. In vivo imaging of lymphatic drainage of cerebrospinal fluid in mouse. Fluids barriers CNS. 2013;10:35.

49. Iliff JJ, et al. A paravascular pathway facilitates CSF flow through the brain parenchyma and the clearance of interstitial solutes, including amyloid beta. Sci Transl Med. 2012;4:147ra111.
50. Weller RO, et al. Lymphatic drainage of the brain and the pathophysiology of neurological disease. Acta Neuropathol. 2009;117:1-14.

51. Walter BA, et al. The olfactory route for cerebrospinal fluid drainage into the peripheral lymphatic system. Neuropathol Appl Neurobiol. 2006;32:388-96.

52. Bacyinski A, et al. The paravascular pathway for brain waste clearance: current understanding, significance and controversy. Front Neuroanat. 2017;11:101.

53. Pollay M. The function and structure of the cerebrospinal fluid outflow system. Cerebrospinal Fluid Res. 2010;7:9.

54. Sakka L, Coll G, Chazal J. Anatomy and physiology of cerebrospinal fluid. Eur Ann Otorhinolaryngol Head Neck Dis. 2011;128:309-16.

55. Hertz L, et al. Astrocytic and neuronal accumulation of elevated extracellular $\mathrm{K}(+)$ with a $2 / 3 \quad \mathrm{~K}(+) / \mathrm{Na}(+)$ flux ratioconsequences for energy metabolism, osmolarity and higher brain function. Front Comput Neurosci. 2013;7:114.

56. Albuquerque FC, et al. Intracranial venous sinus stenting for benign intracranial hypertension: clinical indications, technique, and preliminary results. World Neurosurg. 2011;75:648-52. discussion 592-5

57. Puffer RC, Mustafa W, Lanzino G. Venous sinus stenting for idiopathic intracranial hypertension: a review of the literature. J Neurointerv Surg. 2013;5:483-6.

58. King JO, et al. Manometry combined with cervical puncture in idiopathic intracranial hypertension. Neurology. 2002;58:26-30.

59. Johanson CE, et al. Multiplicity of cerebrospinal fluid functions: new challenges in health and disease. Cerebrospinal Fluid Res. 2008;5:10.

60. Aspelund A, et al. A dural lymphatic vascular system that drains brain interstitial fluid and macromolecules. J Exp Med. 2015;212:991-9.

61. Da Mesquita S, Fu Z, Kipnis J. The meningeal lymphatic system: a new player in neurophysiology. Neuron. 2018;100:375-88.

62. Denniston AK, et al. The ocular glymphatic system and idiopathic intracranial hypertension: author response to "hypodense holes and the ocular glymphatic system". Investig Ophthalmol Vis Sci. 2017;58:1134-6.

63. Iliff JJ, et al. Brain-wide pathway for waste clearance captured by contrast-enhanced MRI. J Clin Investig. 2013;123:1299-309.

64. Jessen NA, et al. The glymphatic system: a beginner's guide. Neurochem Res. 2015;40:2583-99.

65. Glimcher SA, et al. Ex vivo model of cerebrospinal fluid outflow across human arachnoid granulations. Investig Ophthalmol Vis Sci. 2008;49:4721-8.

66. Grzybowski DM, et al. In vitro model of cerebrospinal fluid outflow through human arachnoid granulations. Investig Ophthalmol Vis Sci. 2006;47:3664-72.

67. Kitchen P, et al. Beyond water homeostasis: diverse functional roles of mammalian aquaporins. Biochim Biophys Acta. 2015;1850:2410-21.

68. Klarica M, et al. Effect of head position on cerebrospinal fluid pressure in cats: comparison with artificial model. Croat Med J. 2006;47:233-8.

69. Murtha LA, et al. Cerebrospinal fluid is drained primarily via the spinal canal and olfactory route in young and aged spontaneously hypertensive rats. Fluids Barriers CNS. 2014;11:12.

70. Newborg B. Pseudotumor cerebri treated by rice reduction diet. Arch Intern Med. 1974;133:802-7.

71. Kupersmith MJ, et al. Effects of weight loss on the course of idiopathic intracranial hypertension in women. Neurology. 1998;50:1094-8.

72. Sinclair AJ, et al. Low energy diet and intracranial pressure in women with idiopathic intracranial hypertension: prospective cohort study. BMJ. 2010;341:c2701. 
73. Alperin N, et al. MRI evidence of impaired CSF homeostasis in obesity-associated idiopathic intracranial hypertension. Am J Neuroradiol. 2013;34:29-34.

74. Orefice $\mathrm{G}$, et al. Radioisotopic cisternography in benign intracranial hypertension of young obese women. A seven-case study and pathogenetic suggestions. Acta Neurol. 1992;14:39-50.

75. Glueck CJ, et al. Idiopathic intracranial hypertension, polycysticovary syndrome, and thrombophilia. J Lab Clin Med. 2005; 145:72-82.

76. Chen J, Wall M. Epidemiology and risk factors for idiopathic intracranial hypertension. Int Ophthalmol Clin. 2014;54:1-11.

77. Fraunfelder FW, Fraunfelder FT. Evidence for a probable causal relationship between tretinoin, acitretin, and etretinate and intracranial hypertension. J Neuroophthalmol. 2004;24:214-6.

78. Libien J, et al. Role of vitamin A metabolism in IIH: Results from the idiopathic intracranial hypertension treatment trial. J Neurol Sci. 2017;372:78-84.

79. Libien J, Blaner WS. Retinol and retinol-binding protein in cerebrospinal fluid: can vitamin A take the "idiopathic" out of idiopathic intracranial hypertension?. J Neuroophthalmol. 2007;27:253-7.

80. Berry DC, et al. Retinoic acid upregulates preadipocyte genes to block adipogenesis and suppress diet-induced obesity. Diabetes. 2012;61:1112-21.

81. Bonet ML, et al. Carotenoids and their conversion products in the control of adipocyte function, adiposity and obesity. Arch Biochem Biophys. 2015;572:112-25.

82. Sinclair AJ, et al. Cerebrospinal fluid corticosteroid levels and cortisol metabolism in patients with idiopathic intracranial hypertension: a link between 11beta-HSD1 and intracranial pressure regulation? J Clin Endocrinol Metab. 2010;95:5348-56.

83. Sinclair AJ, et al. Corticosteroids, 11 beta-hydroxysteroid dehydrogenase isozymes and the rabbit choroid plexus. J Neuroendocrinol. 2007;19:614-20.

84. Rauz S, et al. Expression and distribution of the serum and glucocorticoid regulated kinase and the epithelial sodium channel subunits in the human cornea. Exp Eye Res. 2003;77:101-8.

85. Rauz S, et al. Expression and putative role of 11 betahydroxysteroid dehydrogenase isozymes within the human eye. Investig Ophthalmol Vis Sci. 2001;42:2037-42.

86. Ball AK, et al. Elevated cerebrospinal fluid (CSF) leptin in idiopathic intracranial hypertension (IIH): evidence for hypothalamic leptin resistance? Clin Endocrinol. 2009;70:863-9.

87. Klok MD, Jakobsdottir S, Drent ML. The role of leptin and ghrelin in the regulation of food intake and body weight in humans: a review. Obes Rev. 2007;8:21-34.

88. Tartaglia LA, et al. Identification and expression cloning of a leptin receptor, OB-R. Cell. 1995;83:1263-71.

89. Harris RB. Parabiosis between $\mathrm{db} / \mathrm{db}$ and $\mathrm{ob} / \mathrm{ob}$ or $\mathrm{db} /+$ mice. Endocrinology. 1999;140:138-45.

90. Banks WA. The blood-brain barrier as a cause of obesity. Curr Pharm Des. 2008;14:1606-14.

91. Lampl Y, et al. Serum leptin level in women with idiopathic intracranial hypertension. $\mathrm{J}$ Neurol Neurosurg Psychiatry. 2002;72:642-3.

92. Dhungana S, Sharrack B, Woodroofe N. Cytokines and chemokines in idiopathic intracranial hypertension. Headache. 2009;49:282-5.

93. Behbehani R, et al. Is cerebrospinal fluid leptin altered in idiopathic intracranial hypertension? Clin Endocrinol. 2010;72:851-2.

94. Samanci B, et al. Evidence for potential involvement of proinflammatory adipokines in the pathogenesis of idiopathic intracranial hypertension. Cephalalgia. 2017;37:525-31.

95. Beltowski J, et al. Oxidative stress, nitric oxide production, and renal sodium handling in leptin-induced hypertension. Life Sci. 2004;74:2987-3000.
96. Beltowski J, et al. Up-regulation of renal $\mathrm{Na}+, \mathrm{K}+$-ATPase: the possible novel mechanism of leptin-induced hypertension. Pol J Pharmacol. 2004;56:213-22.

97. Toscano V, et al. Cerebrospinal fluid estrone in pseudotumor cerebri: a change in cerebral steroid hormone metabolism? J Endocrinol Investig. 1991;14:81-6.

98. Klein A, et al. Hyperandrogenism is associated with earlier age of onset of idiopathic intracranial hypertension in women. Curr Eye Res. 2013;38:972-6.

99. Stanczyk FZ, Jurow J, Hsing AW. Limitations of direct immunoassays for measuring circulating estradiol levels in postmenopausal women and men in epidemiologic studies. Cancer Epidemiol Biomark Prev. 2010;19:903-6.

100. Kilgore KP, et al. A population-based, case-control evaluation of the association between hormonal contraceptives and idiopathic intracranial hypertension. Am J Ophthalmol. 2019;197:74-9.

101. Lindvall-Axelsson M, Owman C. Changes in transport functions of isolated rabbit choroid plexus under the influence of oestrogen and progesterone. Acta Physiol Scand. 1989;136:107-11.

102. Dutt M, Jialal I. Physiology, adrenal gland, in statpearls. Treasure Island (FL): StatPearls Publishing LLC; 2018.

103. O'Reilly MW, et al. Hyperandrogenemia predicts metabolic phenotype in polycystic ovary syndrome: the utility of serum androstenedione. J Clin Endocrinol Metab. 2014;99: 1027-36.

104. Hornby C, et al. What do transgender patients teach us about idiopathic intracranial hypertension? Neuro-Ophthalmol. 2017; 41:326-9.

105. Park S, et al. Secondary intracranial hypertension from testosterone therapy in a transgender patient. Semin Ophthalmol. 2014;29:156-8.

106. Kapoor KG. Regarding secondary intracranial hypertension from testostereone therapy in a transgender patient. Semin Ophthalmol. 2015;30:241-2.

107. O'Reilly MW, et al. 11-oxygenated C19 steroids are the predominant androgens in polycystic ovary syndrome. J Clin Endocrinol Metab. 2017;102:840-8.

108. Ding EL, et al. Sex differences of endogenous sex hormones and risk of type 2 diabetes: a systematic review and meta-analysis. JAMA. 2006;295:1288-99.

109. Dalmasso C, et al. Cardiometabolic effects of chronic hyperandrogenemia in a new model of postmenopausal polycystic ovary syndrome. Endocrinology. 2016;157:2920-7.

110. Azziz R, et al. Positions statement: criteria for defining polycystic ovary syndrome as a predominantly hyperandrogenic syndrome: an androgen excess society guideline. J Clin Endocrinol Metab. 2006;91:4237-45.

111. Morgan CL, et al. Evaluation of adverse outcome in young women with polycystic ovary syndrome versus matched, reference controls: a retrospective, observational study. J Clin Endocrinol Metab. 2012;97:3251-60.

112. Mani H, et al. Diabetes and cardiovascular events in women with polycystic ovary syndrome: a 20 -year retrospective cohort study. Clin Endocrinol. 2013;78:926-34.

113. Randeva HS, et al. Cardiometabolic aspects of the polycystic ovary syndrome. Endocr Rev. 2012;33:812-41.

114. Canoy D, et al. Fat distribution, body mass index and blood pressure in 22,090 men and women in the Norfolk cohort of the European Prospective Investigation into Cancer and Nutrition (EPIC-Norfolk) study. J Hypertens. 2004;22:2067-74.

115. Yusuf $\mathrm{S}$, et al. Obesity and the risk of myocardial infarction in 27,000 participants from 52 countries: a case-control study. Lancet. 2005;366:1640-9.

116. Grundy SM, Adams-Huet B, Vega GL. Variable contributions of fat content and distribution to metabolic syndrome risk factors. Metab Syndr Relat Disord. 2008;6:281-8. 
117. Hornby $\mathrm{C}$, et al. Evaluating the fat distribution in idiopathic intracranial hypertension using dual-energy X-ray absorptiometry scanning. Neuroophthalmology. 2018;42:99-104.

118. Flexner LB, Winters $H$. The rate of formation of cerebrospinal fluid in etherized cats. Am J Physiol Cell Physiol. 1932;101: $697-710$.

119. Lorenzo AV, Page LK, Watters GV. Relationship between cerebrospinal fluid formation, absorption and pressure in human hydrocephalus. Brain. 1970;93:679-92.

120. Deck MDF, Potts DG. Movements of ventricular fluid levels due to cerebrospinal fluid formation. Am J Roentgenol. 1969;106:354-68.

121. Potts DG, Bergland RM. Roentgenologic studies of cerebrospinal fluid formation in the dog. Am J Roentgenol Radium Ther Nucl Med. 1969;105:756-62.

122. Potts DG, Deck MD, Deonarine V. Measurement of the rate of cerebrospinal fluid formation in the lateral ventricles of the dog. Radiology. 1971;98:605-10.

123. de R, et al. Fluid formed by choroid plexus; a technique for its collection and a comparison of its electrolyte composition with serum and cisternal fluids. J Neurophysiol. 1960;23:485-95.

124. Florez YN, et al. Semiautomatic analysis of phase contrast magnetic resonance imaging of cerebrospinal fluid flow through the aqueduct of Sylvius. Magma. 2006;19:78-87.

125. Lee $\mathrm{JH}$, et al. CSF flow quantification of the cerebral aqueduct in normal volunteers using phase contrast cine MR imaging. Korean J Radiol. 2004;5:81-6.
126. Luetmer $\mathrm{PH}$, et al. Measurement of cerebrospinal fluid flow at the cerebral aqueduct by use of phase-contrast magnetic resonance imaging: technique validation and utility in diagnosing idiopathic normal pressure hydrocephalus. Neurosurgery. 2002;50:534-43. discussion 543-4

127. Jones HC, Deane R, Bucknall RM. Developmental changes in cerebrospinal fluid pressure and resistance to absorption in rats. Brain Res. 1987;430:23-30.

128. Ma Q, et al. Outflow of cerebrospinal fluid is predominantly through lymphatic vessels and is reduced in aged mice. Nat Commun. 2017;8:1434.

129. Roy Chowdhury U, Holman BH, Fautsch MP. A novel rat model to study the role of intracranial pressure modulation on optic neuropathies. PLoS One. 2013;8:e82151.

130. Sanvitto GL, Azambuja NA, Marques M. A technique for collecting cerebrospinal fluid using an intraventricular cannula in rats. Physiol Behav. 1987;41:523-4.

131. Botfield H, et al. Decorin prevents the development of juvenile communicating hydrocephalus. Brain. 2013;136:2842-58.

132. Niemeyer JE. Telemetry for small animal physiology. Lab Anim. 2016;45:255-7.

133. Szuts TA, et al. A wireless multi-channel neural amplifier for freely moving animals. Nat Neurosci. 2011;14:263-9.

134. Guild SJ, McBryde FD, Malpas SC. Recording of intracranial pressure in conscious rats via telemetry. J Appl Physiol. 1985;119:576-81. 2015 